\title{
An Analysis of Dust Halo and Extinction Toward X Persei
}

\author{
Lynne A. Valencic ${ }^{1}$ and Randall K. Smith ${ }^{2}$
}

\begin{abstract}
Interstellar dust grain models are not sufficiently constrained by UV extinction curves to be able to distinguish between them. By testing grain models in the X-ray regime and applying elemental abundance constraints, we show to what extent the models can reproduce the observables in these regimes, and if they are capable of doing so while respecting the abundance limits. We tested the MRN and WD grain models. The fits to the X-ray data do not allow us to distinguish between MRN and WD; both models provide reasonable fits, but cannot do so while respecting the elemental abundance constraints. The situation in the UV regime is similar. Both MRN and WD underestimate the hydrogen column density $\mathrm{N}_{H}$. The model of ZDA provides promising results, as it finds $\mathrm{N}_{H}$ much closer to the UV-measured value; further testing of this model is called for.
\end{abstract}

Subject headings: dust, extinction, $\mathrm{x}$-ray halo, grain models

\section{Introduction}

While interstellar dust is vitally important to the Galaxy, being necessary both in stellar system and molecule formation, the grains' exact nature remains mysterious. Although their effects are seen in almost all astronomical observations, where they both scatter and absorb light in the sight line, they are maddeningly difficult to study. An improved understanding of dust will lead to robust models that can more accurately recover an object's intrinsic spectral energy distribution, and will allow us to probe the grains' environment. Grain models must characterize the composition, morphology, and size distribution of dust grains; these are constrained by elemental abundances and observations of light scattering and absorption (extinction) in the UV/optical regimes and emission in the IR. However, numerous grain models meet the conditions placed upon them by these constraints, and up until now, there

\footnotetext{
${ }^{1}$ NASA/Goddard Space Flight Center, Code 662, Greenbelt, MD 20771; valencic@milkyway.gsfc.nasa.gov

${ }^{2}$ The Johns Hopkins University, Dept. of Physics \& Astronomy, Bloomberg Hall, Baltimore, MD
} 
has been no way to discriminate amongst them. Thus, we must look to other wavelength regimes for further constraints. X-ray dust halos are an excellent, and mostly untapped, diagnostic of grain characteristics and can provide badly needed constraints on grain models.

As noted by Overbeck (1965) and Hayakawa (1970), dust grains scatter X-rays into a halo around a point source according to the size and position of the grains and the energy of the incident photons. Several authors have observed halos and tried to use them to measure properties of the ISM. Catura (1983) confirmed the predictions of Overbeck and Hayakawa by using Einstein Observatory's HRI instrument to observe dust halos around 6 point sources to find the $\mathrm{H}$ column density $N_{H}$ and grain size and mass distributions. The radial size of the halos ranged from 60 " to 600 ", so the large end of the size distribution, which effects angles < 100", were not well sampled. They found that the scattering dust grains ranged from $0.03 \mu \mathrm{m}-1.0 \mu \mathrm{m}$, roughly with the range considered in the model of Mathis, Rumpl, \& Nordsieck (1977; hereafter MRN). Since the size of the halos was restricted to large. angles, a contribution from grains larger than $1.0 \mu \mathrm{m}$, which would be seen at angles smaller than Catura considered, could not be ruled out. In 1986, Mauche \& Gorenstein followed up on Catura's work by obtaining complementary data from Einstein Observatory's IPC instrument, which was more sensitive and had greater energy resolution than the HRI, making it more suited to observing faint, extended halos. These data were used to test three different grain size distirubtions, that of MRN, Oort-van de Hulst (1946), and grains of a single size; it was concluded that while the observed halos ruled out the possibility of a single size distribution, they could not distinguish between the MRN or the Oort-van de Hulst distributions to describe the halos. Mathis et al. (1995) used ROSAT observations of Nova Cygni 1992 to examine the composition and "fluffiness" of dust grains, while using optical extinction measurements as a guide. They found that in order for there to be sufficient optical extinction without overly strong $x$-ray halos, the grains had to be largely vaccuum; however, a study of the dust along the line of sight toward another X-ray source, GX 13+1, did not confirm this need for fluffy grains (Smith, Edgar, \& Shafer 2002).

Grain models have evolved greatly over the past three decades since their inception with MRN. In the MRN model, which was designed to model extinction using solar reference abundances, a simple power law size distribution with sharp cutoffs at $50 \AA$ and $0.25 \mu \mathrm{m}$ was considered for bare spherical silicate and graphite grains. While MRN adequately modeled the extinction, it did not account for any of the features of the ISM attributed to very small grains or molecules such as the extended red emission in the optical band, the unidentified IR bands, or the thermal emission from very small grains that have been subject to singlephoton heating. Further, it did not account for any processing which is likely to affect grains such as shocks and irradiation. Despite this, MRN is still often used in ISM studies, and so will be considered here. Others have since improved upon MRN, adding optical 
constants for "astronomical" silicates and graphitic carbon and extending the size range to about that of large molecules, $30 \AA$ (Draine \& Lee 1984; Draine \& Anderson 1985). In 1990, Désert, Boulanger, \& Puget constructed a model which included polycyclic aromatic hydrocarbon (PAH) molecules to account for observed emission from the near- to far-IR. Their work emphasized the important role that molecules and very small grains must play in a successful model. The importance of non-spherical, porous grains was shown by Fogel \& Leung (1998), who found that fractal grains could account for extinction while using about $30 \%$ less mass than the traditional spherical grains; this is an important conclusion in light of the fact that elemental abundances are often the most difficult constraint for grain models to meet. Weingartner \& Draine (2001; hereafter WD) further improved their model by including PAHs and very small carbonaceous grains with laboratory-based optical constants, in addition to astronomical silicates; their size distribution was very broad, ranging from 3.5 $\AA$ to about $1.0 \mu \mathrm{m}$, but had difficulty meeting the abundance requirements. In 2004, Zubko, Dwek, \& Arendt (hereafter ZDA) did a comprehensive comparison of several models using silicates, carbonaceous grains, composites, PAHs, and ices by simultaneously fitting diffuse ISM extinction $\left(R_{V}=3.1\right)$, IR emission, and abundance requirements. Their results showed that several models can adequately describe optical extinction and IR emission while staying within the bounds of the constraints. This highlights the need to bring more constraints to bear upon the models.

In this paper, we will do this by combining for the first time X-ray halo data from XMM-Newton with UV/optical extinction observations for line of sight through the diffuse ISM and elemental abundance measurements. The ideal place to start is a reddened object with both strong X-ray emission and UV flux; the star X Per is perfect for this.

\section{The X Per System}

$\mathrm{X}$ Per (HD 24534) has long been known to be a Be/neutron star binary (i.e. Rappaport $\&$ van den Heuvel 1982). The orbital parameters are not known (Telting et al. 1998), but since it is in the Be/X-ray class of High Mass X-Ray Binaries, it can be assumed that its components have a wide orbit and do not interact, so that the material accreted onto the neutron star comes entirely from the capture of the stellar wind of the Be star, as opposed to Roche Lobe overflow (Nagase 1989). The optical component has been classified by Lesh (1968) as O9.5pe and Slettebak (1982) as O9.5IIIe, but other workers have found it to be B0e (Mendoza 1958; Lyubimkov et al. 1997; Roche et al. 1997). The neutron star companion has a spin period of $13.9 \mathrm{~min}$ (White et al. 1976). Some (Hutchings et al. 1974; Kemp \& Barbour 1983) have found an orbital period of $580 \mathrm{~d}$, though these results have not been 
confirmed (Larionov \& Larionova 1989; Roche et al. 1993). X Per's V band magnitude varies from about 6.1 to 6.8 , and work by Roche et al. (1993) show a wide range of variability from the optical to NIR, which is unrelated to the X-ray brightness (Mavromatakis 1993; Haberl 1994) and is most likely due to the formation and dissipation of a circumstellar disk. The system's geometry is believed to be pole-on, rather than equator-on, so emission should play a larger role than absorption; indeed, the bright phases are redder than the fainter, bluer phases and so the "disk-free" state corresponds to the faint phase of the light curve (Roche et al. 1993). The true photometry of the star can be observed at that point. During the disk-free phase, the star may be considered non-variable (Roche et al. 1993).

\section{Theory of Dust Halos}

X-rays scatter off of dust grains as a function of grain size, distance from source, and incident photon energy. An illustration is shown in Fig. 1. X-rays are observed at an angle $\theta_{\text {obs }}$ after being scattered at an angle $\theta_{\text {sca }}$ and travel a distance $d$ from the scattering particle to the observer, who is at a distance $D$ from the source. The quantity $\theta_{\text {sca }}$ is sufficiently small that the small angle approximation applies (Mathis \& Lee 1991), and so $\theta_{o b s} / \theta_{s c a}=1-x$, where $x=d / D$. At low optical depths $(\tau<1.3)$, single scattering dominates the halo, while at higher optical depths, multiple scattering becomes important (Mathis \& Lee 1991). Since the X Per line of sight is dominated by the diffuse interstellar medium (see the next section), we are confident that multiple scattering is negligible. Assuming single scattering, the halo intensity as a function of angular distance from the source $\theta, I(\theta)$, can be found for each energy bandpass (Mathis \& Lee 1991):

$$
I(\theta)=N_{H} F_{X} \int S(E) d E \int f(a) d a \int \frac{g(x)}{(1-x)^{2}} \frac{d \sigma_{s c a}(E, \theta, a, x)}{d \Omega} d x
$$

where $N_{H}$ is the total hydrogen column density, $F_{X}$ is the X-ray source flux, $f(a) d a$ is the number of dust grains per $\mathrm{H}$ atom over the interval a to $a+d a, S(E) d E$ is the X-ray spectral energy distribution, normalized to unity, and $f(x)$ is the hydrogen density at a distance $x D$ relative to the average hydrogen density for the given sight line. For a smooth distribution, $g(x) \equiv 1 ;$ for a cloud at a distance $x_{0}, g(x)=\delta\left(x-x_{0}\right)$. The quantity $d \sigma / d \Omega$ is the scattering cross section of the grain, which is a function of the incident $X$-ray energy, grain size, and position. The quantity $d \sigma / d \Omega$ can be found either by the Rayleigh-Gans (RG) approximation or an exact Mie calculation. The $R G$ approxmimation requires two assumptions: first, that there is no reflection off of the grain, and second, that the incident wave does not undergo a phase change after it enters the grain. Mie theory calculates the exact scattering of the 
incident wave and does not assume that all scattering sites will add coherently. While the result is exact for spherical grains only, it describes scattering off non-spherical particles well to first-order (Bohren \& Huffman 1998). In 1998, Smith \& Dwek found that the RG approximation fails at energies less than $1 \mathrm{keV}$, substantially overestimating the scattering cross section, and thus the halo intensity. They recommended using the exact Mie solution for energies less than $2 \mathrm{keV}$.

\section{Data Analysis}

\subsection{X-ray Halo}

On 25 Feb. 2003, X Per was observed with the XMM-Newton EPIC-MOS instrument for $31.4 \mathrm{ksec}$ in full-frame mode. Datasets for both X Per and Mkn 421 (which would be used to determine the instrument's point spread function, or PSF) were downloaded from the HEASARC data archive and reprocessed using the XMM-Newton Science Analysis System (SAS) version 6.5.0. Information about the XMM data used is in Table 2. An EPIC-MOS image can be seen in Fig. 5 .

For the EPIC-MOS camera, an event is identified when there is a local signal enhancement in flat fielded images that is above a defined energy threshold and that forms a predefined pattern. There are 32 patterns, divided into groups according to how many pixels are involved: singles, doubles, triples, and quadruples. Singles and doubles are considered the highest quality, as these are compact, with the highest charge at the center, and all other surrounding pixels are below the threshold. For more than one pixel to be affected by an incoming X-ray, either the X-ray hit near a pixel edge, or electrons released by a high-energy photon diffused into neighboring pixels. Testing has shown that low-energy ("soft") X-rays tend to produce the first 13 patterns. These 13 are the standard set of valid X-ray events and are well calibrated.

We began by making filtered event lists for both X Per and Mkn 421 by applying the standard filters to remove invalid events (i.e. events with patterns outside the aforementioned 13) and observations made during periods of high background. Extraneous sources were also removed; this was done by searching the Simbad database for any other X-ray sources in our images and removing them (Costantini, Freyberg, \& Predehl 2005). There were 10 sources in our X Per data and 13 in Mkn 421. Not all were detected in the images, but those positions were nonetheless removed from consideration.

In Fig. 5, it can be seen that the inner regions of $X$ Per have low flux; this is due to pileup, where multiple $\mathrm{X}$-rays are registered in one pixel or neighboring pixels before they 
can be cycled out. The result is that numerous events are treated as one event with the sum of the incident photon energies, leading to a much harder spectrum, since the soft X-rays are undercounted and shifted to higher energies. In order to assess the severity of the pileup, SAS procedures were used to plot the observed single and double event distributions and compare them to the modeled event distributions. There were significant discrepancies, as would be expected for a heavily piled up image, so the inner 40 " region was excised from the event list. The resulting event list was then rechecked using the same prodecures. The single and double event distributions from the "clean" list fit the model distributions, so we believe the effects of pileup have been removed from our data. In addition to the central 40 ", the transfer streak was also removed.

Concentric annuli centered on the source and evenly spaced in log radius were applied to the images and used to extract the radial profiles from 40" to 600", over the energy bands $1.1-3.8 \mathrm{keV}$, in bins of $0.3 \mathrm{keV}$. Exposure maps were then made for each energy level. The same annuli were applied to the exposure maps. By dividing the radial profiles with their corresponding effective areas and exposure times from the exposure maps, the radial profiles in units of photons $\mathrm{s}^{-1} \mathrm{~cm}^{-2} \operatorname{arcmin}^{-2}$ for each energy level were found.

Once the radial profiles were found, the instrumental contributions (PSF and background) needed to be assessed and removed. XMM-Newton's PSF has been modeled as an energy-dependent King profile (Ghizzardi 2002). However, optically thin lines of sight do not have halos and can also be used to determine the PSF. Costantini et al. (2005) showed that there is excellent agreement between the XMM-Newton's modeled PSF and Mkn 421's radial profile, and so we treat Mkn 421 as a halo-free source and fit it to determine the PSF. To do this, Mkn 421's surface brightness profile was fitted for each energy level extracted. It was determined that a broken power law provided the best fits for $\mathrm{E}<2.0 \mathrm{keV}$; for higher energies, a power law provided the best fit. An example fit is shown in Fig. 2. These functions were then scaled and fitted for each energy level of the X Per profiles, along with a constant background (Bocchino et al. 2005; Costantini et al. 2005). With the instrumental contributions accounted for in each profile, the remainder of the flux was the halo. This was fitted with the haloes produced by the MRN and WD grain models assuming either a smooth or single-cloud dust distribution. Values of $\mathrm{N}_{H}$ (and cloud location, when appropriate) were found for each energy level.

\subsection{Extinction Curves}

The main grain diagnostic and model constraint until now has been provided by UV extinction curves. These are made by pair method (Massa, Savage, \& Fitzpatrick 1983). 
Reddened spectra are divided by unreddened spectra of similar spectral type, so that the stellar features and continua are removed and only the effects of interstellar dust remain. The resulting curve shows the wavelength-dependent extinction along a sight line, and is normalized to either $\mathrm{E}(\mathrm{B}-\mathrm{V})$ or $\mathrm{A}_{V}$. In 1989, Cardelli, Clayton, \& Mathis (CCM) found that the curves depend only on one parameter, the ratio of total-to-selective extinction $R_{V}$ $\left(=\mathrm{A}_{V} / \mathrm{E}(\mathrm{B}-\mathrm{V})\right)$; this was confirmed by Valencic, Clayton, \& Gordon (2004), who found that virtually all Galactic lines of sight conform to this law. $R_{V}$ is itself is a general indicator of grain coagulation in the Galaxy, as lines of sight which pass through dense media have higher values of $\mathrm{R}_{V}$ than those which pass through more diffuse media. $\mathrm{R}_{V}$ typically ranges from 2.5 to 5.5 , with an average diffuse ISM value of 3.1.

Large aperture, low dispersion spectra was obtained for X Per from the Final Archive at the Multimission Archive at Space Telescope (MAST). Datasets were selected to allow examination of the spectra at both bright and faint phases of the light curve. The shorter wavelengths are less affected than the longer ones, with the flux ratio for $1 / \lambda>4 \mu \mathrm{m}^{-1}$ $(\lambda<2500 \AA)$ being about $90 \%$. For $3.3 \mu \mathrm{m}^{-1}<1 / \lambda<4 \mu \mathrm{m}^{-1}(2500 \AA<\lambda<3300 \AA)$, the ratio decreases to about $80 \%$. However, even in that wavelength range, the bright and faint phase fluxes are still within $3 \sigma$ of each other. Nonetheless, the dataset corresponding to the disk-free phase was selected to examine the interstellar extinction and was used in conjunction with the disk-free phase optical photometry of Kharchenko (2001) to find the reddening, $\mathrm{E}(\mathrm{B}-\mathrm{V})$. The spectra were compared to the dereddened UV spectra from Cardelli, Sembach, \& Mathis (1992), until the best spectral match (HD 36512; B0V) was found. The area around the Lyman $\alpha$ feature was discarded between 8.0 and $8.4 \mu \mathrm{m}^{-1}$. The spectral types, photometry, sources, and reddening are in Table 1 . Values of $\mathrm{R}_{V}$ and $\mathrm{A}_{V}$ were also found. The usual formula for $A_{V}$ requires knowledge of an object's near-IR photometry (Fitzpatrick 1999); while such data exists for X Per, it has been noted by others that its JHK photometry is variable and the uncertainties on JHK in the faint phase are somewhat large $(\sim 0.1 \mathrm{mag})$. Thus, $\mathrm{A}_{V}$ was deduced from $\mathrm{R}_{V}$, and checked with Hipparcos parallax and JHK photometry for general accuracy. To determine $R_{V}$, the extinction curve was fit with the CCM extinction law until $\chi^{2}$ was minimized. This yielded $\mathrm{R}_{V}^{\text {fit }}$ of 3.06 , and $\mathrm{A}_{V}^{\text {fit }}$ = 1.19. Using the Hipparcos parallax (1.21 mas), the BOV absolute magnitude $\mathrm{M}_{V}=-4.0$ of Schmidt-Kaler (1982) and the disk-free photometry, a reassuringly identical value for $A_{V}$ was obtained. Lastly, using Norton et al.'s (1991) JHK photometry during a disk-free phase, we found $\mathrm{R}_{V}=3.02 \pm 0.10$, in good agreement with our best-fit value. Table 1 summarizes the results.

We extended the extinction curve into the FUV using low resolution archived FUSE spectra. Since the IUE comparison star, HD 36512, has not been observed with FUSE, another B0V, HD 97471, was used. X Per and HD 97471 were observed with FUSE on 
September 12, 2000 for $6.4 \mathrm{ksec}$ and March 24, 2000 for $4.2 \mathrm{ksec}$, respectively. They were reprocessed with the most recent version of CalFUSE (v. 3.1) before their placement in the archive, so subsequently we have not re-reduced them. For each star, the different FUSE detector segments were corrected for channel drift by cross-correlating and shifting them to a common wavelength scale, then co-added. The spectra range from $8.4-10.5$ $\mu \mathrm{m}^{-1}$. Absorption due to interstellar hydrogen at these wavelengths is very strong, and the spectra had to be reconstructed in order to recover the continua (Rachford et al. 2001, 2002; Cartlegde et al. 2005; Sofia et al. 2005) and find the extinction curve. Following the techniques previous workers, the conintua for both X Per and HD 97471 were modeled with low order polynomials. Frémat et al.'s (2002) online catalog, which is based on Morton's (1991) line list, was used to identify molecular hydrogen lines. These were fitted with Voigt profiles with IRAF routines, and the comparison spectrum was dereddened with standard software. The spectra were rebinned to $5 \AA$, the resolution of IUE. Stellar photospheric lines were not removed, as these are cancelled by the comparison spectra. The spectrum was recovered up to $1 / \lambda \cong 9.4 \mu \mathrm{m}^{-1}$ and the extinction curve was found and merged with the longer-wavelength curve, with a smooth transition from the IUE to FUSE curve at $8.4 \mu^{-1}$. The final IUE+FUSE curve was then rechecked and found to be consistent with the $\mathrm{R}_{V}=$ 3.06 curve found previously, in agreement with Sofia et al. (2005). The entire UV spectrum, with IUE and FUSE data, is shown in Fig. 3. The full curve, overplotted with the $\mathrm{R}_{V}=$ 3.06 curve, is shown in Fig. 4.

\subsection{Grain Models}

The observed extinction may be thought of as the sum over the extinction contributed by $j$ dust components, each of which has a characteristic chemical compostion and grain size distribution, $f^{j}(a)$, which is the number of grains per $\mathrm{H}$ atom over the radius interval $a$ to $a+d a$. It may be calculated by solving

$$
\tau(\lambda)=N_{H} \sum_{j=1}^{n} \int_{a_{\min }}^{a_{\max }} \pi a^{2} Q_{e x t}^{j}(\lambda, a) f^{j}(a) d a
$$

where $\mathrm{N}_{H}$ is the hydrogen column density for a given sight line, $a$ is the grain radius, and $Q_{e x t}^{j}(\lambda, a)$ is the grain's extinction efficiency factor (Kim, Martin, \& Hendry 1994; ZDA).

The MRN and WD models were considered in this work. The MRN grain size distribution function is simply $f(a) \propto a^{-3.5}$ for $50 \AA<a<0.25 \mu \mathrm{m}$. Numerous workers have investigated and built upon this model (e.g. Draine \& Lee 1984; Kim, Martin, \& Hendry 
1994), which was originally designed to fit only the UV extinction caused by the diffuse interstellar medium with bare, spherical graphite and silicate grains. It is an unphysical model; it has very sharp grain size cutoffs, and it excludes the very small grains (VSGs) and large molecules (such as polycyclic aromatic hydrocarbons, or PAHs) which give rise to the observed IR and microwave emission (Léger \& Puget 1984; Draine \& Lazarian 1998). Despite this, it is still often used in studies of the ISM, and so it is considered here.

In contrast, WD includes VSGs and has a smoother, more realistic exponential cutoff, with grain sizes ranging from $3.5 \AA<a<0.8 \mu \mathrm{m}$. It is also far more complex, having 11 adjustable parameters, and tends to require more elemental material than is available. The function itself depends on the grain sizes and compositions. For silicate grains,

$$
\frac{f^{s}}{n_{H}}=\frac{C_{s}}{a}\left(\frac{a}{a_{t, s}}\right)^{\alpha_{s}} F\left(a ; \beta_{s}, a_{t, s}\right) G\left(a ; a_{t, s}, a_{c, s}\right)
$$

where

$$
F\left(a ; \beta_{s}, a_{t, s}\right)= \begin{cases}1+\beta a / a_{t, s} & : \quad \beta \geq 0 \\ \left(1-\beta a / a_{t, s}\right)^{-1} & : \quad \beta<0\end{cases}
$$

and

$$
G\left(a ; \beta_{s}, a_{t, s}\right)= \begin{cases}1 & : 3.5 \AA<a<a_{t, s} \\ \exp \left[\left(a_{t, s}-a\right) / a_{c, s}\right]^{3} & : \quad a>a_{t, s}\end{cases}
$$

For carbonaceous grains,

$$
\frac{f^{g}}{n_{H}}=K(a)+\frac{C_{g}}{a}\left(\frac{a}{a_{t, g}}\right)^{\alpha_{g}} F\left(a ; \beta_{g}, a_{t, g}\right) G\left(a ; a_{t, g}, a_{c, g}\right)
$$

where the functions $F$ and $G$ are the same as for silicates, but with the carbon variables, and $K(a)$ describes the population of VSGs, as found by Li \& Draine (2001):

$$
\frac{K(a)}{n_{H}}=\sum_{j=1}^{2} \frac{L_{j}}{a} \exp \left\{\frac{-1}{2}\left[\frac{\ln \left(a / a_{0, j}\right)}{\sigma}\right]^{2}\right\}
$$

where 


$$
L_{j}=\frac{3}{(2 \pi)^{3 / 2}} \frac{\exp \left(-4.5 \sigma^{2}\right)}{\rho \sigma a_{0, j}^{3}} \frac{b_{C, j} m_{C}}{1+\operatorname{erf}(\eta)}
$$

and

$$
\eta=\frac{1}{\sqrt{2}}\left[3 \sigma+\frac{1}{\sigma} \ln \left(\frac{a_{0, j}}{3.5 \AA}\right)\right]
$$

In Eqn. (7), $\rho$ is the density of a graphitic grain, $m_{C}$ is the mass of a carbon atom, and $b_{C}$ is the carbon abundance taken up by very small grains (VSG). Following WD, we let $b_{C, 1}=0.75 b_{C}, b_{C, 2}=0.25 b_{C}, a_{0,1}=3.5 \AA, a_{0,2}=30 \AA$, and $\sigma=0.4$. WD found good fits to an $\mathrm{R}_{V}=3.1$ curve for cases with different values of $b_{C}$, ranging from $b_{C}=0$ to $b_{C}=6.0$ $\times 10^{-5}$. Models show that the microwave emission can be described with $b_{C}=2-4 \times 10^{-5}$ (Draine \& Li 2001), while the diffuse Galactic emission can be reproduced when $b_{C}=4$ $6 \times 10^{-5}$ ( Li \& Draine 2001). WD produced fits for all these cases, but that in which $b_{C}=$ $6.0 \times 10^{-5}$ was recommended. In all cases, $a_{c, s}=0.1$. Thus, for our fits, we also hold $b_{C}=$ $6.0 \times 10^{-5}$ and $a_{c, s}=0.1$.

The most recent dielectric function calculations for carbonaceous grains and "smoothed astronomical" silicates were obtained from Dr. Bruce T. Draine's website (http: //www.astro. princeton.edu / draine/) and used for both models. They are very similar to those of Draine \& Laor (1993) over UV wavelengths; the main difference is the excision of a feature near 6.5 $\mu \mathrm{m}^{-1}$ in the silicate dielectric function and the redistribution of the oscillator strengths from $8-10 \mu \mathrm{m}^{-1}$. These functions were extended into the X-ray regime (Draine 2003). Over the region $0.1-10 \mu \mathrm{m}^{-1}$, the dielectric functions are the same as those used in WD.

Wiscombe's Mie code $(1979,1980)$ was used to calculate the extinction coefficients $Q_{e x t}$ and the scattering cross sections $d \sigma / d \Omega$; representative values of $Q_{a b s}$ and $Q_{s c a}$ are shown in Fig. 6 for different grain compositions and sizes. We assumed the grains were spherical and homogeneous. Since graphite is a strongly anisotropic material, its dielectric function depends on the orientation of the electric field. The standard " $\frac{1}{3}-\frac{2}{3}$ " approximation for graphite (i.e. $Q_{e x t}^{g r}=\frac{1}{3} Q_{e x t}^{g r}\left(\epsilon_{\|}\right)+\frac{2}{3} Q_{e x t}^{g r}\left(\epsilon_{\perp}\right)$ was used, where $\epsilon_{\|}\left(\epsilon_{\perp}\right)$ is the component of the graphite delectric tensor for the electric field parallel (perpendicular) to the the crystal axis (Draine \& Malhotra 1993). 


\subsection{Elemental Abundances}

Elemental abundances are the most challenging constraint for grain models to meet. By determining how much of an element is available in the ISM, and how much is in the gas phase (either by measurement or assumption), one can easily find how much is in the solid phase. Over the years, B stars, young F and G stars, and the Sun have all been used as the cosmic yardstick, but there are large differences amongst these, and the measurements have large uncertainties. Sofia \& Meyer (2001) determined that B star metallicities are not accurate representations for the ISM, and recommended that either young F/G stars or solar values be used, as these tended to be consistent with each other. However, in the latest measurement of solar abundances, the $\mathrm{C} / \mathrm{H}$ has dropped precipitously, from a value consistent with that of young $F$ and $G$ stars to a value that is within the uncertainty of $B$ star carbon abundances ("old" solar: $3.63 \times 10^{-4}$ (Anders \& Grevesse 1989, hereafter AG89); "new" solar: $2.453( \pm 0.30) \times 10^{-4}$ (Asplund, Sauval, \& Grevesse 2005, hereafter ASG05); young F/G stars: $3.58( \pm 0.82) \times 10^{-4}$ (Sofia \& Meyer 2001); B stars: $1.90( \pm 0.77) \times 10^{-4}$ (Sofia \& Meyer 2001)). ASG05's abundances for silicon and iron are also somewhat lower, though the decreases are not as dramatic; they are still within the young F/G star uncertainties (Sofia \& Meyer 2001).

In light of the fluctuating solar values and concern over whether the Sun is indeed a valid yardstick for the ISM, we have found the Si, Fe, and C abundances needed for the models using both the solar abundances of $\mathrm{AG} 89$, with $\mathrm{Si} / \mathrm{H}=3.55 \times 10^{-5}, \mathrm{Fe} / \mathrm{H}=3.467 \times 10^{-5}$, and $\mathrm{C} / \mathrm{H}=3.63 \times 10^{-4}$, and $\mathrm{ASG} 05$, with $\mathrm{Si} / \mathrm{H}=3.245 \times 10^{-5}, \mathrm{Fe} / \mathrm{H}=2.818 \times 10^{-5}$, and $\mathrm{C} / \mathrm{H}$ $=2.45 \times 10^{-4}$. Abundances from AG89 are comparable to those of F and G stars. Further, Sofia (1998) measured the $\mathrm{C}$ gas phase abundance along this line of sight to be $1.06 \times 10^{-4}$, so that the amount of carbon available in the solid phase may be further constrained. Both Si and $\mathrm{Fe}$ are assumed to be $100 \%$ depleted into dust. The grain densities were assumed to be $2.2 \mathrm{~g} \mathrm{~cm}^{-3}$ for carbonaceous grains and $3.3 \mathrm{~g} \mathrm{~cm}^{-3}$ for silicates. The mean molecular mass of silicate was assumed to be consistent with that of $\mathrm{MgFeSiO}_{4}$, or 172 amu. Using the abundances of AG89, these led to total available volumes of $3.079 \times 10^{-27} \mathrm{~cm}^{3} \mathrm{H}^{-1}, 3.001 \times 10^{-27}$ $\mathrm{cm}^{3} \mathrm{H}^{-1}$, and $2.336 \times 10^{-27} \mathrm{~cm}^{3} \mathrm{H}^{-1}$ for $\mathrm{Si}, \mathrm{Fe}$, and $\mathrm{C}$, respectively. Using ASG05, these numbers become $2.811 \times 10^{-27} \mathrm{~cm}^{3} \mathrm{H}^{-1}, 2.439 \times 10^{-27} \mathrm{~cm}^{3} \mathrm{H}^{-1}$, and $1.264 \times 10^{-27} \mathrm{~cm}^{3} \mathrm{H}^{-1}$, respectively.

\section{Method and Results}

The haloes at $\mathrm{E}=1.1,1.4,1.7,2.0$, and $2.3 \mathrm{keV}$ were fitted with MRN and WD grain models. One energy band, $1.7 \mathrm{keV}$, was selected for further investigation; this band was 
chosen as the halo appeared strong in that band and the results from the MRN and WD fits were typical of those of the group overall. The measured extinction was fitted with both models. Standard Fortran integration and $\chi^{2}$ minimization codes were used. We began with the extinction by finding the grain radius at which $\chi^{2}$ was minimized and from that, the requisite abundances. This was straightforward for MRN, but for WD, the optimal parameter values had to be found first. This was done by first varying $\alpha_{g}$ and $\alpha_{s}$, then $\beta_{g}$ and $\beta_{s}$, then $a_{t, g}$ and $a_{t, s}$, then $a_{c, g}$, and finally $C_{g}$ and $C_{s}$; the new values are listed in Table 3. A comparison of grain size distribution functions can be seen in Fig. 7. Fits to the extinction can be seen in Fig. 8 and Fig. 9. The abundances required by the fits are listed in Table 4.

The haloes were fit four times for each grain model. First, the maximum grain radii were held constant at their standard values and $\mathrm{N}_{H}$ was found, then $\mathrm{N}_{H}$ was held at its UV-measured value of $2.19 \times 10^{21} \mathrm{~cm}^{-2}$ (Diplas \& Savage 1994; Rachford et al. 2002; Cunningham, McCray, \& Snow 2004) and the maximum grain radii were found. These are referred to as Set 1 and Set 2, respectively. This was repeated for smooth and single cloud dust distributions. In the single cloud case, the location of the cloud along the line of sight was also calculated. The required abundances were then found for each fit and compared to the (solar abundance - gas phase abundance) and that which is needed to produce the UV extinction. The grain models, values of $N_{H}$, required abundances, and cloud positions $\mathrm{x}_{0}$ (when appropriate) are listed in Table 5 for Set 1 and Table 6 for Set 2. Values of $\chi^{2}$ are given as $\log \left[\left(I_{\text {mod }}-I_{o b s}\right)^{2} / \sigma^{2}\right]$. Fig. 10 and Fig. 11 show the best fits to the halo using both a smooth and single cloud distributions for the MRN and WD models, respectively.

\section{Discussion}

In the UV, as seen in Figs. 8 and 9, both models produce generally reasonable fits to the data. WD produces a much tighter fit to the $2175 \AA$ bump than MRN, which slightly underestimates the extinction from about $4-6.5 \mu \mathrm{m}^{-1}$; at the same time, WD slightly underestimates the amount of FUV extinction, though the difference does not extend beyond $2 \sigma$. MRN tends to slightly overestimate the extinction between 6.5 and $8 \mu \mathrm{m}^{-1}$. While both models tend to overestimate the extinction between 3 and $4 \mu \mathrm{m}^{-1}$, they are still at or within $1 \sigma$ of the data.

Perhaps the most striking thing about Figs. 10 and 11 is the relatively good fits to the data, regardless of dust distribution or the grain model. Both models were able to reproduce the halo well, consistent with previous findings (Xiang et al. 2005). Only the single-cloud WD distribution yields fits with notably higher $\chi^{2}$ values in Sets 1 and 2 . With those 
exceptions, the fits' $\chi^{2}$ values are closely clustered. In both Sets 1 and 2 , the single-cloud WD fit has the largest $\chi^{2}$ value. The trouble the code had in fitting this model can also be seen in the values of $\mathrm{x}_{0}$ it found, placing the cloud very close to the Sun $\left(\mathrm{x}_{0} \sim 0.0\right)$. The difficulty was not unique to this energy band; at low energies $(E=1.1,1.4,1.7 \mathrm{keV}) \mathrm{x}_{0}$ tended toward 0 for this model. The average $\mathrm{x}_{0}$ over all 5 bands was only $\mathrm{x}_{0}^{\text {ave }}=0.19 \pm 0.28$. Because of the large $\chi^{2}$, this fit is not considered any further.

The single-cloud MRN fits are better in relation to the rest of their sets, and have the benefit of requiring elemental abundances more in line with that of extinction. Indeed, in Set 2 , it was found that the required abundances needed to fit the halo when $N_{H}$ was fixed at the UV-measured value was the same as that needed to fit the UV extinction. However, they, like the single-cloud WD fits, place the cloud disturbingly close to the Sun for this energy band. Again, these low values of $x_{0}$ most likely reflect the difficulty the fitting code had with fitting a single cloud to the data for this energy level, even though $x_{0}$ is not as obviously spurious as for single-cloud WD. It is worth noting that when $x_{0}$ values for Set 1-single-cloud MRN were averaged over the energy bands, a much more reasonable value of $\mathrm{x}_{0}^{a v e}=0.40 \pm 0.22$ was found. The $\mathrm{x}_{0}$ found for Set 1 , single-cloud MRN for $\mathrm{E}=1.7 \mathrm{keV}$ falls outside the uncertainty range, further suggesting that the Set $2 \mathrm{x}_{0}$ may not truly represent $\mathrm{x}_{0}$ along this line of sight.

The smooth distibutions were able to reproduce the halo just as well, and in some cases better than, the single-cloud distributions. This disagrees with the findings of Xiang et al. (2005), who concluded that the majority of scattering grains for several strong X-ray point sources are near the source. The smooth distributions tended to require higher abundances than single-cloud distributions. In Sets 1 and 2, MRN needs amounts that do not agree with the extinction or solar limits. The result is similar for smooth distribution WD in Set 1 . In Set 2 , however, WD requires abundances that are only somewhat higher than that of the extinction.

Both models require copious amounts of $\mathrm{Si}, \mathrm{Fe}$, and $\mathrm{C}$ to fit the extinction; this has been noted before by numerous workers (Kim, Martin, \& Hendry 1994; WD; ZDA; Li 2005). Even with the relatively generous abundances of AG89, neither model can fit the extinction and remain comfortably within the abundance constraints, though MRN comes close, requiring only slightly more than what is available for Si. Not surprisingly, things get worse when the abundances of AGS05 are considered, with the requirements for $\mathrm{Si}$ and Fe increasing to nearly the limit, and $\mathrm{C}$ jumping to almost twice as much as is available. The situation for WD is no better; while it requires only $\sim 90 \%$ of available $\mathrm{C}$ from AG89, it is over the limit for $\mathrm{Si}$ and $\mathrm{Fe}$ by $\sim 17 \%$ and $20 \%$, respectively, and cannot meet any of the constraints of ASG05. 
Even higher elemental abundances are typically needed to fit the haloes. The single cloud distributions tend to require lower amounts than smooth distributions, but even these either approach or exceed the constraints. In Set 1, MRN needs about $15 \%$ more $\mathrm{C}$ than provided with AG89; in Set 2, this rises to $20 \%$ for the smooth distribution. In both sets, WD requires more $\mathrm{Si}$ and $\mathrm{Fe}$ than MRN, needing virtually $100 \%$ or more $\mathrm{Si}$ and Fe than is available with AG89.

Clearly, the new solar abundances pose a great challenge to the models, raising the specter of another elemental "budget crisis" (Kim \& Martin 1996). The existence of porous dust has been suggested as a way around this (Mathis 1996; Snow \& Witt 1996; Fogel \& Lenung 1998) but previous searches for it have not been fruitful (Smith, Edgar, \& Schafer 2002). Further, Dwek (1997) showed by analyzing the predicted IR emission and UV extinction that porous grains cannot be a major component to the ISM, and Li (2005) concluded that porous grains cannot solve the problem with the new solar abundances without assuming extremely oblate or prolate shapes. Dwek (1997) also listed several ways out of the budget crisis, without resorting to porous grains, emphasizing the inhomogeneity of the ISM, both in space and time, and the importance of fractionization effects in star formation. In light of this, it is possible that the abundance requirements yielded by AG89 may more accurately represent the true depletions for this line of sight.

Overall, both models tend to underestimate $\mathrm{N}_{H}$, WD more so than MRN, consistent with other observations (Smith, Edgar, \& Schafer 2002; Xiang, Zhang, \& Yao 2005). This is true of both smooth and single cloud distributions, but it is particularly noticeable in the single cloud case, as can be seen in Table 7 . There is much variation across the energy levels, with standard deviations up to $60 \%$ of the average $\mathrm{N}_{H}$ per model and dust distribution. In order reduce this spread, we have also calculated the average $\mathrm{N}_{H}$ by removing values of $\mathrm{N}_{H}$ which fall outside of 1 standard deviation. These new $N_{H}$ are listed in Table 7 in parentheses. In general, they are slightly higher than the original values but remain low; indeed, only the smooth distribution MRN fit yielded an average $N_{H}$ that was within the uncertainty of the UV-measured value, despite the new, "cleaned up" average $\mathrm{N}_{H}$ values. WD produces lower values of $\mathrm{N}_{H}$ than MRN because its grain size distribution includes more large grains, leading to more total scattering cross section per $\mathrm{H}$ atom (Xiang et al. 2005).

As a final test, we derived $\mathrm{N}_{H}$ using the model of ZDA, as can be seen in Table 8. ZDA tested 15 models of varying compostions using the the method of regularization to solve for the grain size distribution. We used the "BARE-GR-S" formulation. Both the smooth and single cloud distributions produce higher $\mathrm{N}_{H}$ - within $1 \sigma$ of the UV-measured value - than the other two models with a comparable amount of spread. The single-cloud distribution yielded $x_{0}^{\text {ave }}=0.47 \pm 0.22$, which is similar to that found for MRN ( $x_{0}^{a v e}=0.40$ ), and much 
larger than the result from WD ( $\mathrm{x}_{0}^{\text {ave }}=0.19 \pm 0.28$ ). There was good agreement between the average values of $\mathrm{N}_{H}$ found with both the smooth and single-cloud distributions and the UV-measured $N_{H}$, although as with MRN and WD, there were large variations between results from different energy bands.

Regarding MRN and WD, the goodness of the fits did not allow us to distinguish if one or the other was bettter at modeling haloes. A denser line of sight, with a correspondingly stronger halo, may allow for such discrimination; however, UV observations are not possible for very dense sight lines, so any sight lines selected for such a multiwavelength study must be chosen with care.

\section{Conclusions}

Our conclusions are as follows:

1) Both MRN and WD had trouble reproducing the haloes and the extinction at the same time. The abundances needed to fit the haloes typically were larger than that needed for the extinction, leading to poor fits for the extinction. However, both models provided good fits to the halo; the data for this line of sight do not allow us to distinguish between them.

2) Neither MRN nor WD could fit the extinction or the haloes without violating abundance constraints. This was especially true when the B-star-like ASG05 values were considered; in that case, the models needed up to twice the abundances as was available. The problem is lessened, though not fully alleviated, when old solar values (which are roughly equivalent to those of $\mathrm{F}$ and $\mathrm{G}$ stars) are used.

3) While both models find $\mathrm{N}_{H}$ similar to the UV for the energy band $\mathrm{E}=1.7 \mathrm{keV}$, they underestimate $\mathrm{N}_{H}$ on average. WD does so to a greater extent than MRN, and the single-cloud distributions for both models do so more than the smooth distributions. The ZDA model, in contrast, found an average $N_{H}$ that was in better agreement with the UV value than MRN or WD. Further investigations involving this model are called for.

\section{REFERENCES}

Anders, E. \& Grevesse, N. 1989, Geochim. Cosmochim. Acta, 53, 197.

Asplund, M., Sauval, J., \& Grevesse, N. 2005, ASP Conference Series, vol. 336, 25. 
Bocchino, F., van der Swaluw, E., Chevalier, R. \& Bandiera, R. 2005, A\&A, 442, 539

Bohren \& Huffman 1983

Cardelli, J., Clayton, G. C. , \& Mathis, J. S. 1989, ApJ, 345, 245

Cardelli, J. A., Sembach, K. R. \& Mathis J. S. 1992, AJ, 104, 1916

Cartledge, S. I. B. et al. 2005, ApJ, 630, 355

Catura, R. C. 1983, ApJ, 275, 645

Costantini, E., Freyberg, M. J., \& Predehl, P. 2005, A\&A, 444, 187

Cunningham, N., McCray, R., \& Snow, T. P. 2004, ApJ, 611, 353

Désert, F.-X., Boulanger, F., \& Puget, J. L. 1990, A\&A, 237, 215

Diplas, A. \& Savage, B. D. 1994, ApJS, 93, 211

Draine, B. T. 2003, ApJ, 598, 1026

Draine, B. T. \& Anderson, N. 1985, ApJ, 292, 494

Draine, B. T. \& Lazarian, A. 1998, ApJ, 494, 19

Draine, B. T. \& Lee, H. M. 1984, ApJ, 285, 89

Draine, B. T. \& Malhotra, S. 1993, ApJ, 414, 632

Dwek, E. 1997, ApJ, 484, 779

Fitzpatrick, E. 1999, PASP, 111, 63

Fogel, M. E. \& Leung, C. M. 1998, ApJ, 501, 175

Frémat, Y. et al. 2002, A\&A, 385, 986

Haberl, F. 1994, A\&A, 283, 175

Hayakawa, S. 1970, Progress of Theoretical Physics, vol. 43, No. 5

Hutchings, J. B., Crowley, A. P., Crampton, D., \& Redman, R. O. 1974, ApJ, 191, L101

Kemp, J. C. \& Barbour, M. S. 1983, ApJ, 264, 237

Kim, S.-H. \& Martin, P. G. 1996, ApJ, 462, 296 
Kim, S.-H., Martin, P. G. \& Hendry, P. D. 1994, ApJ, 422, 164

Kharchenko 2001

Laor, A. \& Draine, B. T. 1993, ApJ, 402, 441

Larionova, L. V. \& Larionov, V. M. 1989, Afz, 31, 421

Léger, A. \& Puget, J. L. 1984, A\&A, 137, 5

Lesh, J. R. 1968, ApJS, 17, 371

Li, A. 2005, ApJ, 622, 965

Li, A. \& Draine, B. T. 2001, ApJ, 554, 778

Lyubimkov, L. S., Rostopchin, S. I., Roche, P., \& Tarasov, A. E. 1997, MNRAS, 285, 549

Mathis, J. S. 1996, ApJ, 472, 643

Mathis, J. S. \& Lee, C.-W. 1991, ApJ, 376, 490

Mathis, J. S., Rumpl, W., \& Nordsieck, K. H. 1977, ApJ, 217, 425

Mathis, J. S., Cohen, D., Finley, J. P., \& Krautter, J. 1995, ApJ, 449, 320

Mauche, C. W. \& Gorenstein, P. 1986, ApJ, 336, 843

assa, D., Savage, B.D., \& Fitzpatrick, E. L. 1983, ApJ, 266, 662

Mavromatakis, F. 1993, A\&A, 276, 353

Mendoza V., E. E. 1958, ApJ, 128, 207

Meyer, D. M., Lauroesch, J. T., Heiles, C., Peek, J.E.G., \& Engelhorn, K. 2006, ApJ, 650, 67

Morton, D. C. 1991, ApJS, 77, 119

Nagase, F. 1989, PASJ, 41, 1

Norton et al. 1991, MNRAS, 253, 579

Overbeck, J. W. 1965, ApJ, 141, 864

Rachford et al. 2001, ApJ, 555, 839 
Rachford et al. 2002, ApJ, 577, 221

Rappaport, S. \& van den Heuvel, E.P.J. 1982, IAUS, 98, 327

Redfield, S. \& Linsky, J. L. 2004, ApJ, 613, 1004

Roche et al. 1993, A\&AS, 97, 277

Roche et al. 1997, A\&A, 322, 139

Slettebak, A. 1982, ApJS, 50, 55

Smith, R.K. \& Dwek, E. 1998, ApJ, 503, 831

Smith, R.K., Edgar, R. J. \& Schafer, R. A. 2002, ApJ, 581, 562

Snow, T. P. \& Witt, A. N. 1996, ApJ, 468, 65

Sofia, U.J., Fitzpatrick, E., \& Meyer, D. M. 1998, ApJ, 504, L47

Sofia, U.J. \& Meyer, D. M. 2001, ApJ, 554, 221

Sofia et al. 2005, ApJ, 625, 167

Valencic, L.A., Clayton, G.C., Gordon, K.D. 2004, ApJ, 616, 912

Weingartner, J. C. \& Draine, B. T. 2001, ApJ, 548, 296

Wiscombe 1979

Wiscombe 1980

White, N.E., Mason, K.O., Sanford, P.W., \& Murdin, P. 1976, MNRAS, 176, 201

Xiang, J., Zhang, S. N. \& Yao, Y. 2005, ApJ, 628, 769

Zubko, V., Dwek, E., \& Arendt R. G. 2004, ApJS, 152, 211

This preprint was prepared with the AAS IATEX macros v5.0. 
Table 1: X Per's UV and Optical Analysis

\begin{tabular}{lllllllll}
\hline \hline LWP & SWP & $\mathrm{B}$ & $\mathrm{V}$ & $\begin{array}{l}\text { Spectral } \\
\text { Type }\end{array}$ & $\begin{array}{l}\text { Comparison } \\
\text { Type }\end{array}$ & $\mathrm{E}(\mathrm{B}-\mathrm{V})$ & $R_{V}$ & $A_{V}$ \\
\hline 31580,31885 & 56062,56583 & 6.87 & 6.78 & O9.5pe, B0e & $\mathrm{B} 0 \mathrm{~V}$ & 0.39 & 3.06 & 1.19 \\
31967 & 56789 & $(0.00)$ & $(0.01)$ & & & $(0.04)$ & & $(0.06)$ \\
\hline
\end{tabular}

Optical photometry from Kharchenko (2001).

Table 2: XMM Dataset Parameters

\begin{tabular}{llllll}
\hline \hline Object & Obs No. & $\begin{array}{l}\text { Exp. Time } \\
(\mathrm{ks})\end{array}$ & M1 Mode & M2 Mode & PN Mode \\
\hline Mkn 421 & 0136541101 & 11.4 & FF-TN & FF-TN & LW-ME \\
X Per & 0151380101 & 31.7 & FF-ME & FF-ME & FF-ME \\
\hline
\end{tabular}

Table 3: WD Best Fit Parameters to X Per

\begin{tabular}{ccccccccccc}
\hline \hline $\mathrm{b}_{C}$ & $\mathrm{C}_{s}$ & $\mathrm{a}_{t s}$ & $\mathrm{a}_{c s}$ & $\alpha_{s}$ & $\beta_{s}$ & $\mathrm{C}_{g}$ & $\mathrm{a}_{t g}$ & $\mathrm{a}_{c g}$ & $\alpha_{g}$ & $\beta_{g}$ \\
\hline $6 \times 10^{5}$ & $1 \times 10^{-13}$ & 0.155 & 0.1 & -2.21 & 0.41 & $9.29 \times 10^{-12}$ & 0.012 & 0.392 & -1.52 & -0.20 \\
\hline
\end{tabular}

Table 4: Percent Silicon, Fe, and Carbon Abundances Required By Extinction

\begin{tabular}{lccccccc}
\hline \hline & \multicolumn{3}{c}{ \% Abundance } & & \multicolumn{3}{c}{ \% Abundance } \\
\cline { 2 - 4 } \cline { 6 - 8 } & $\mathrm{Si}$ & $\mathrm{Fe}$ & $\mathrm{C}$ & & $\mathrm{Si}$ & $\mathrm{Fe}$ & $\mathrm{C}$ \\
\hline$\tau^{M R N}$ & 79.76 & 81.83 & 100.36 & & 87.39 & 100.67 & 185.54 \\
$\tau^{W D}$ & 116.52 & 119.55 & 89.26 & & 127.67 & 147.08 & 164.96 \\
\hline
\end{tabular}

The percent elemental abundance required for the best fits to the extinction, assuming solar abundances of AG89 or ASG05. Both assume the C gas phase measurement of Sofia (1998). 


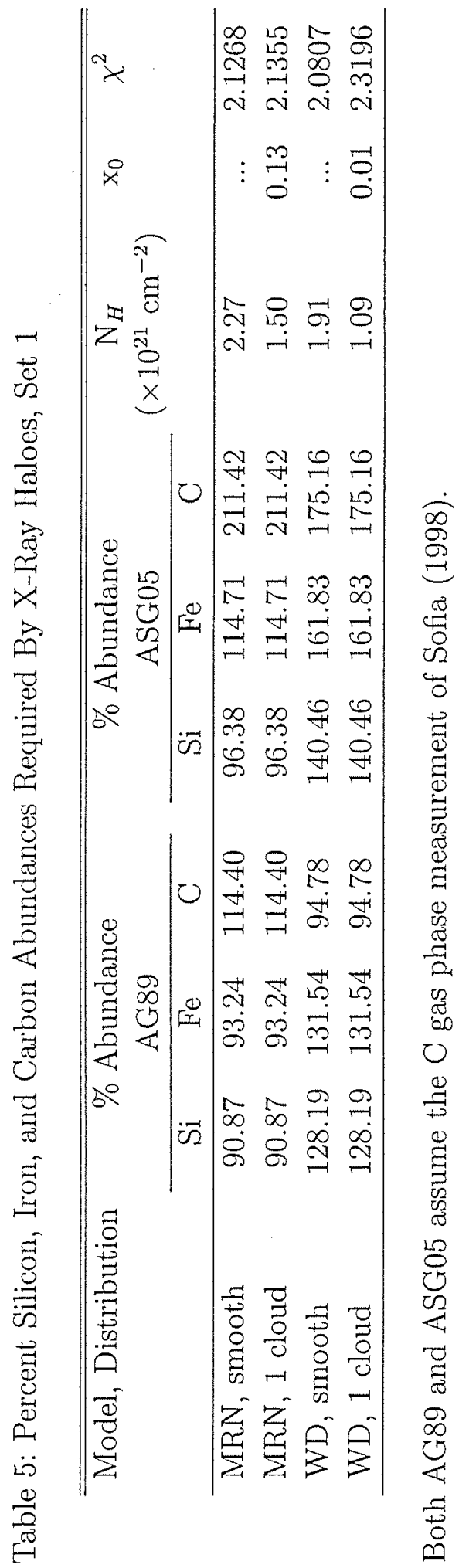




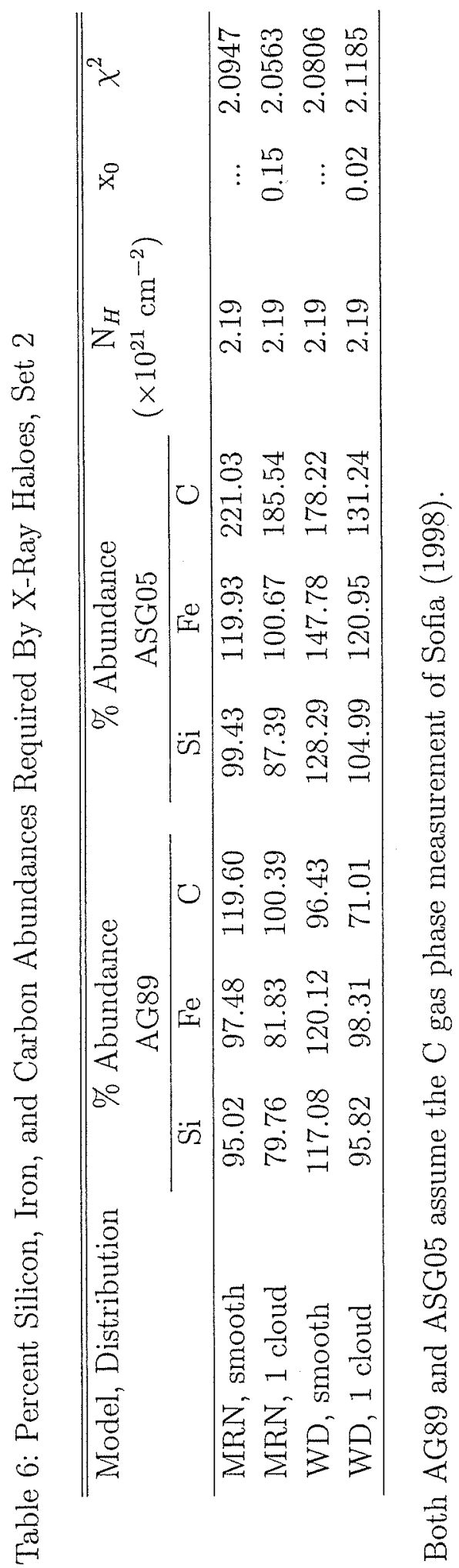




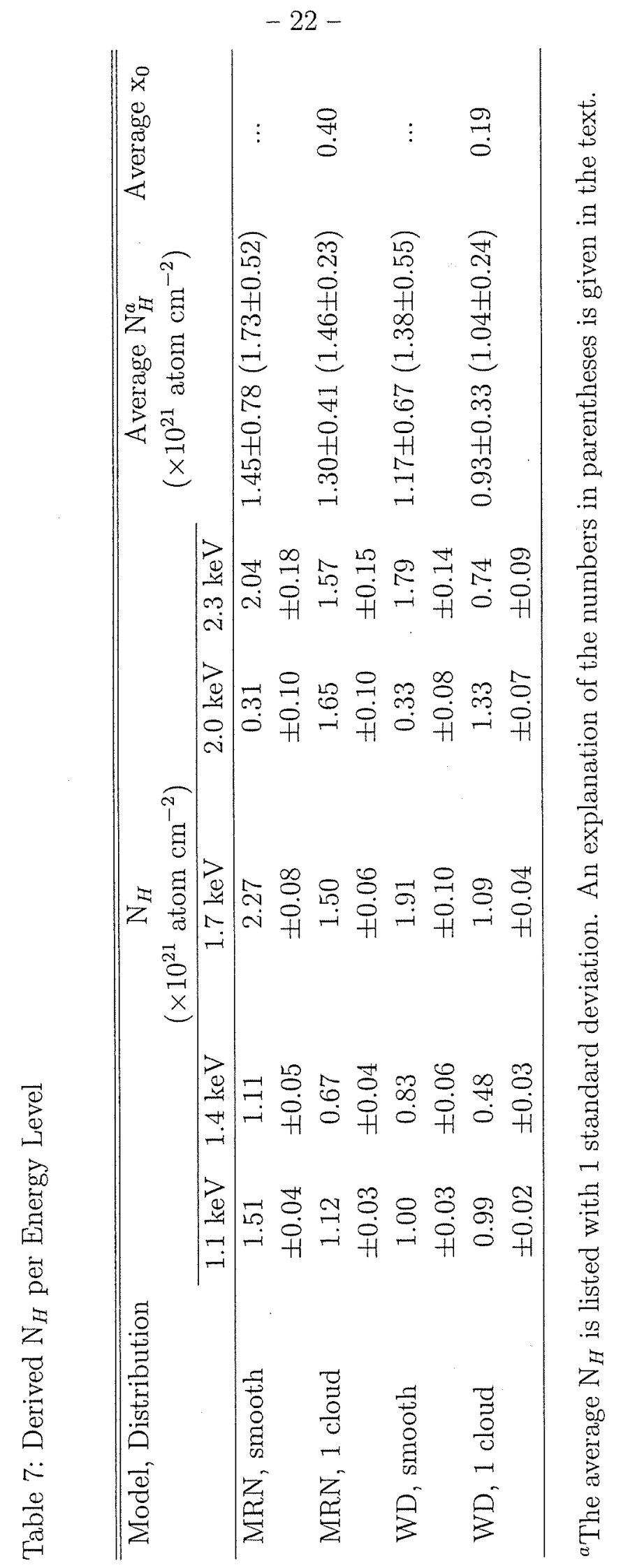




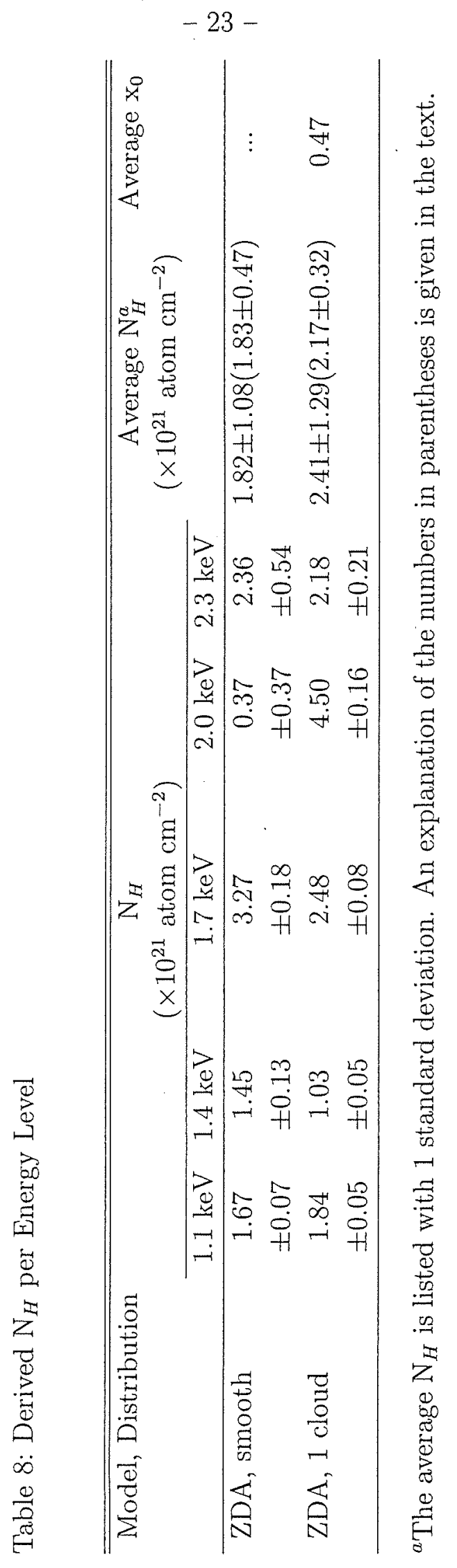




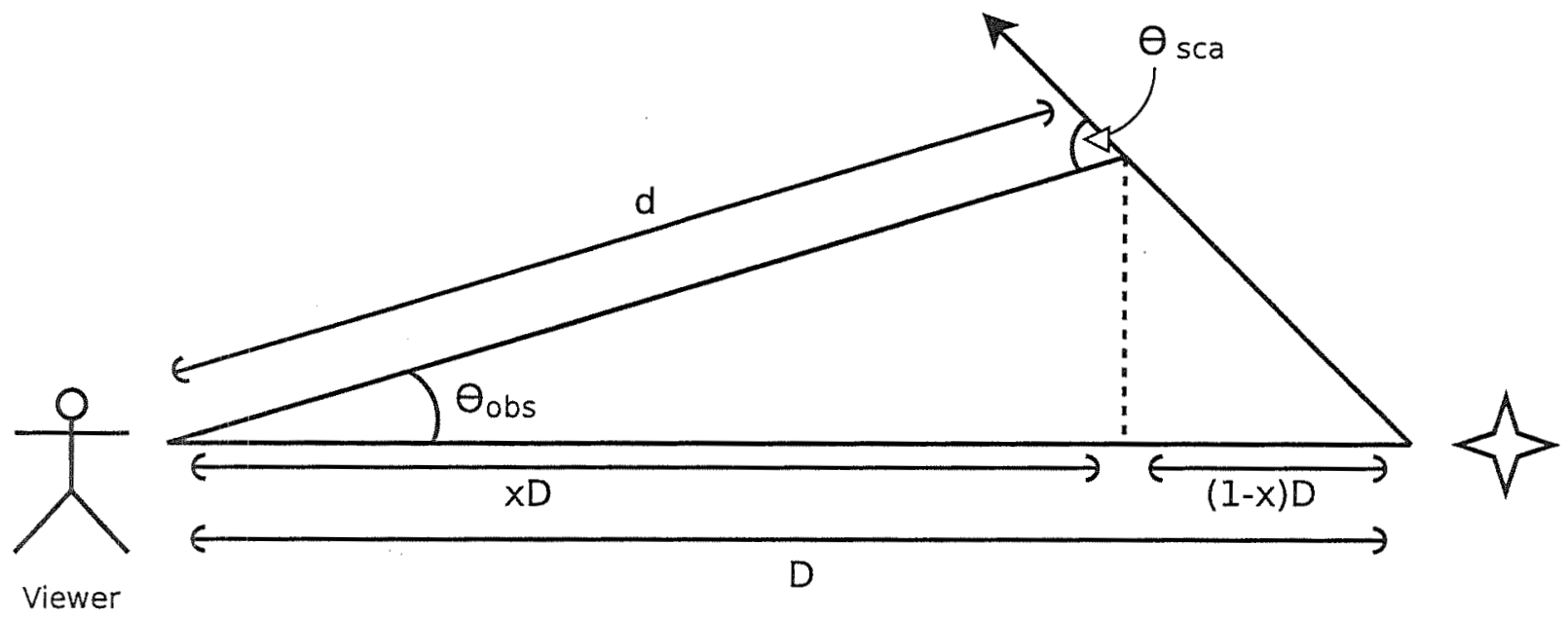

Fig. 1.- An illustration of scattering. The observer is located at $x_{0}=0$ and the source is at $\mathrm{x}_{0}=1$. 


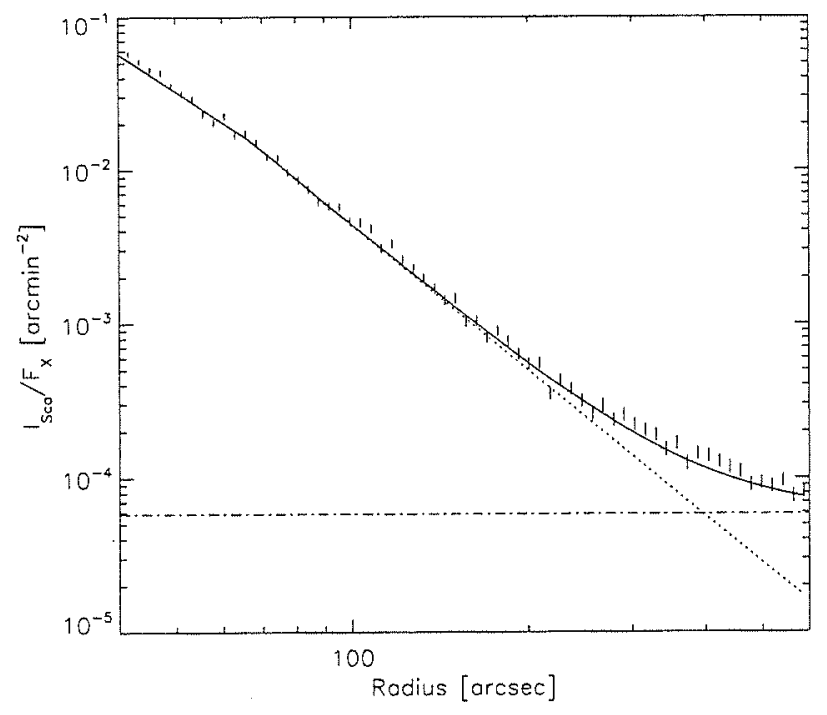

Fig. 2.- Mkn 421, in the $1.7 \mathrm{keV}$ band, fitted with a broken power law (dotted line) and constant background (dot-dash line).

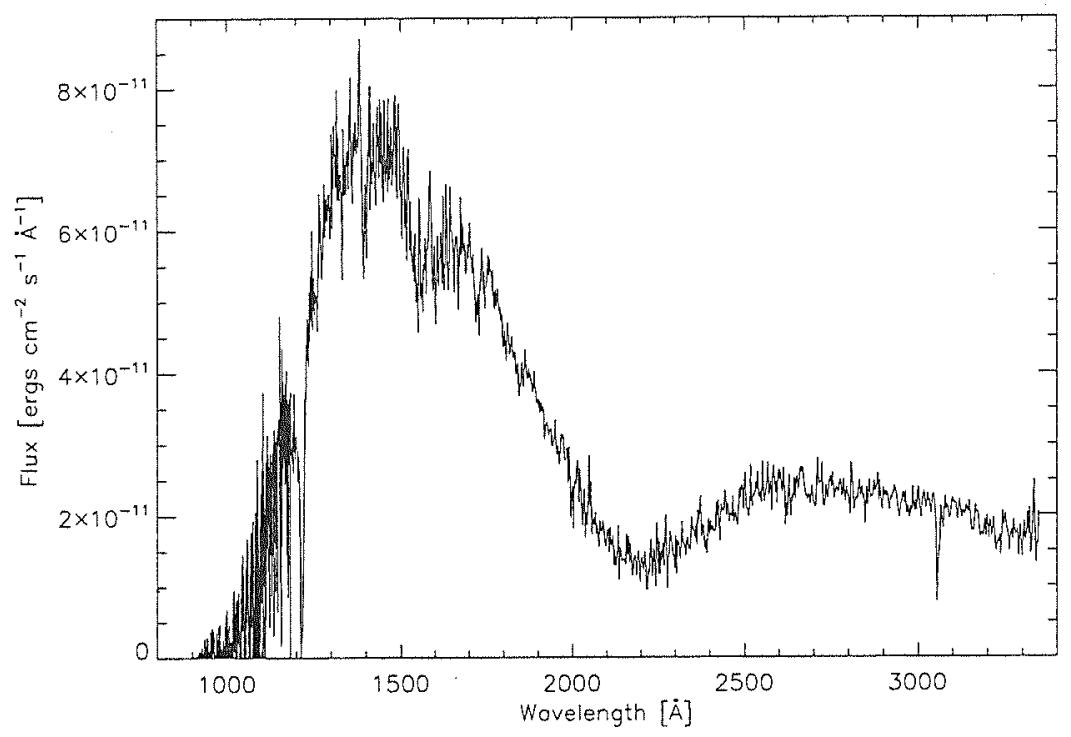

Fig. 3.- UV spectrum of X Per. 


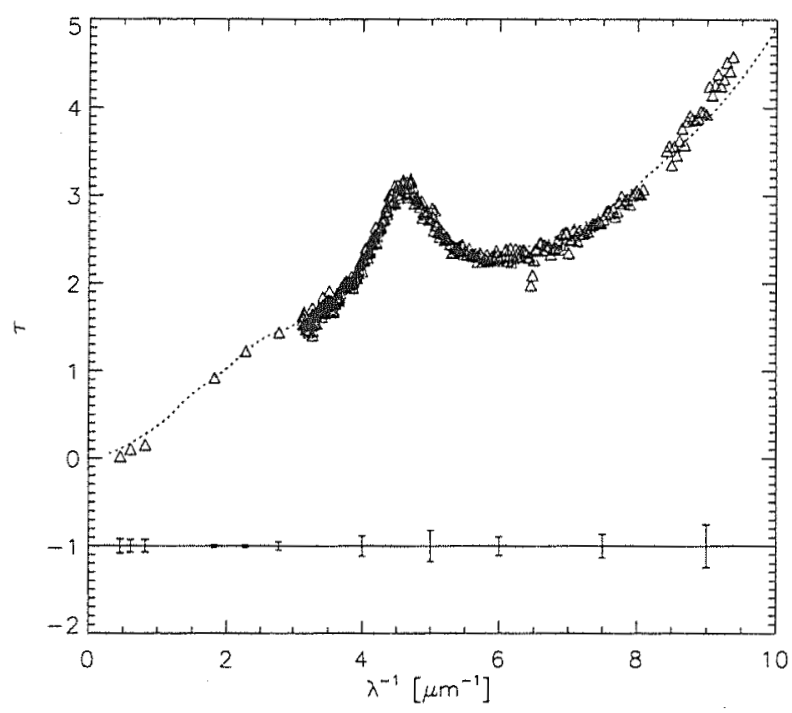

Fig. 4.- Triangles: measured optical depth. Dotted line: the best fit CCM curve $\left(\mathrm{R}_{V}=\right.$ 3.06). Representative error bars are shown.

Fig. 5.- Image of X Per taken with XMM-Newton's EPIC camera. The characteristic pile-up effect of diminishing counts is visible at the center of the object. The transfer streak is also visible, as are several spurious detections of other objects in the field. 


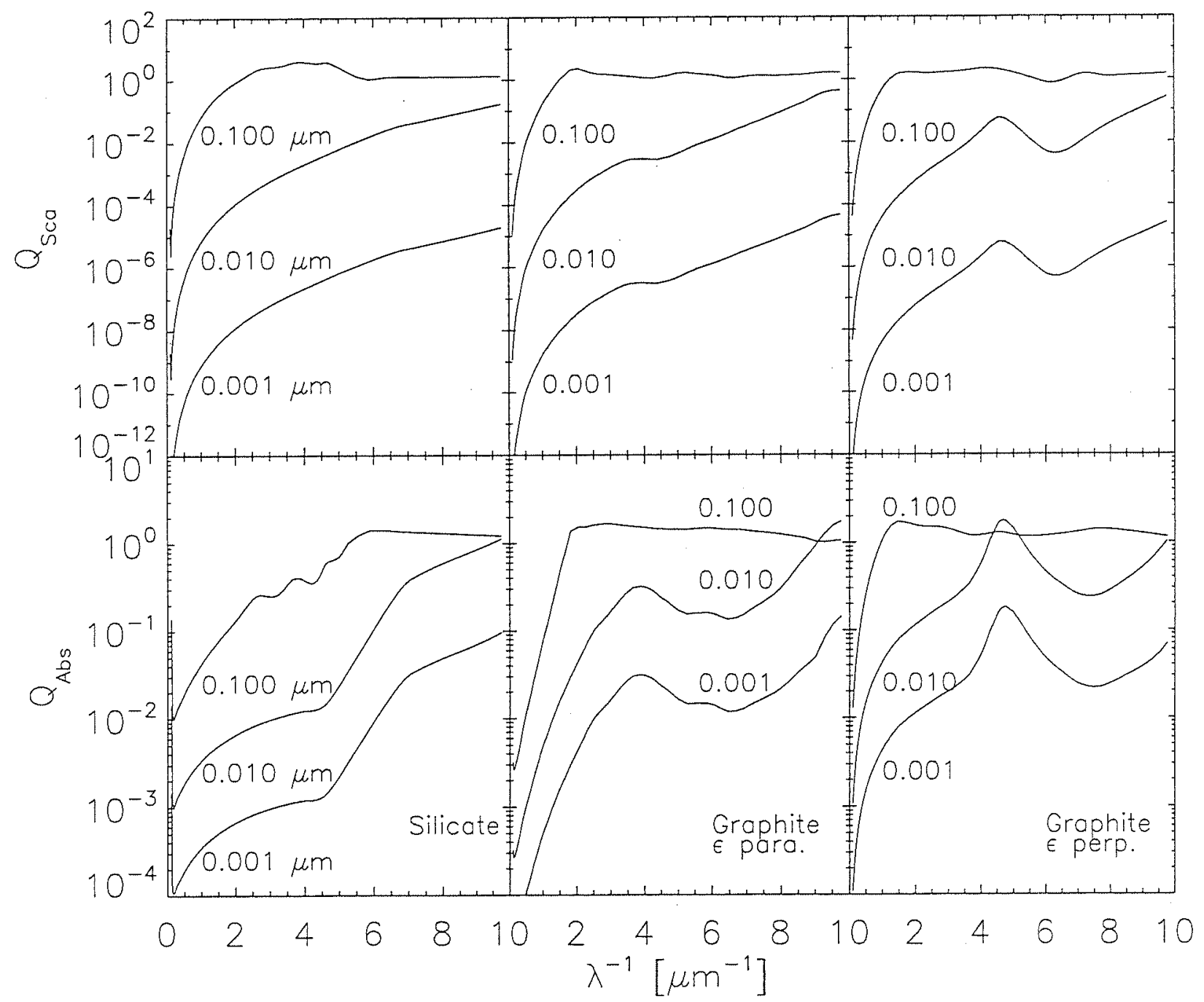

Fig. 6.- The scattering and absorption coefficients for silicates and graphitic carbon for the grain sizes indicated. 


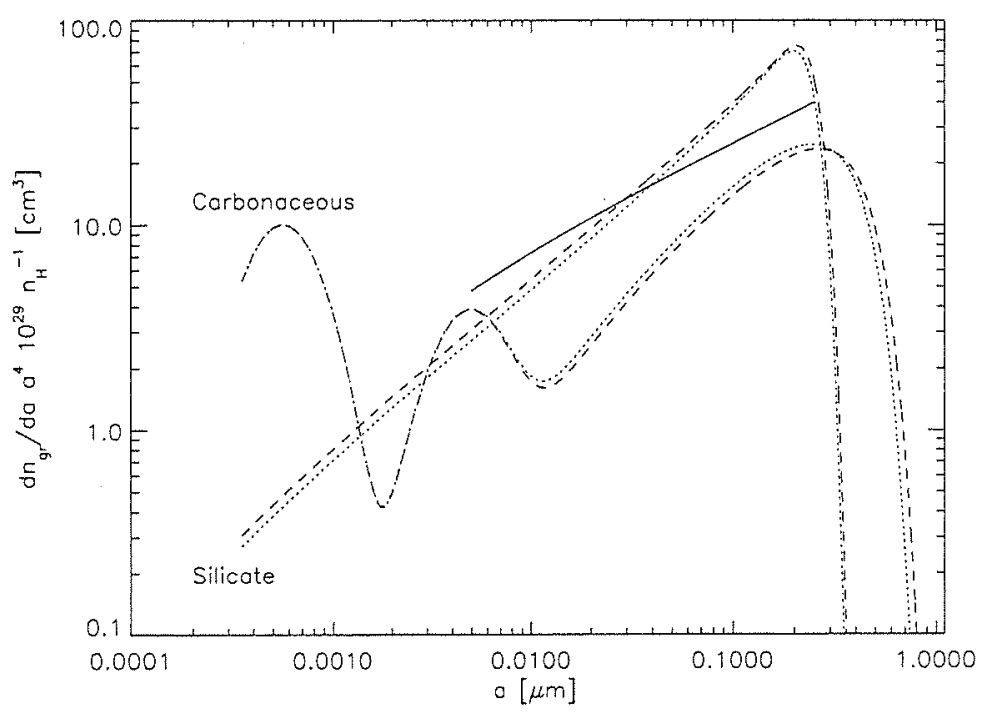

Fig. 7.- A comparison of grain size distrubutions. Solid line: MRN (both silicates and carbonaceous grains). Dashed line: WD model for $R_{V}=3.1$, with $b_{C}=6 \times 10^{-5}$. Dotted line: best fit to $\mathrm{X}$ Per extinction with $b_{C}=6 \times 10^{-5}$.

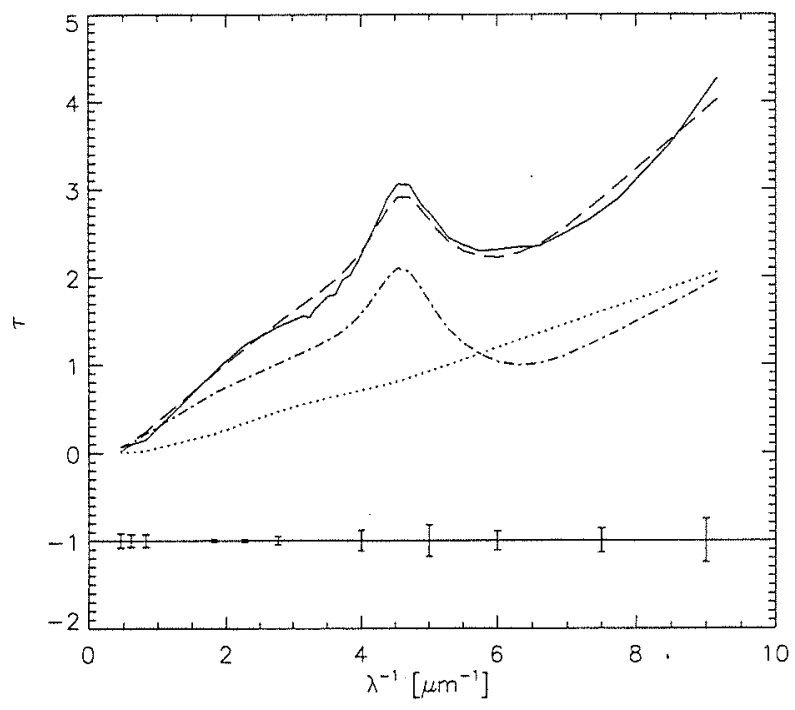

Fig. 8.- The fit to the extinction data (solid line) using the MRN model (long dash line). The dotted and dot-dash lines are the silicate and graphitic components, respectively. Representative error bars are shown. 


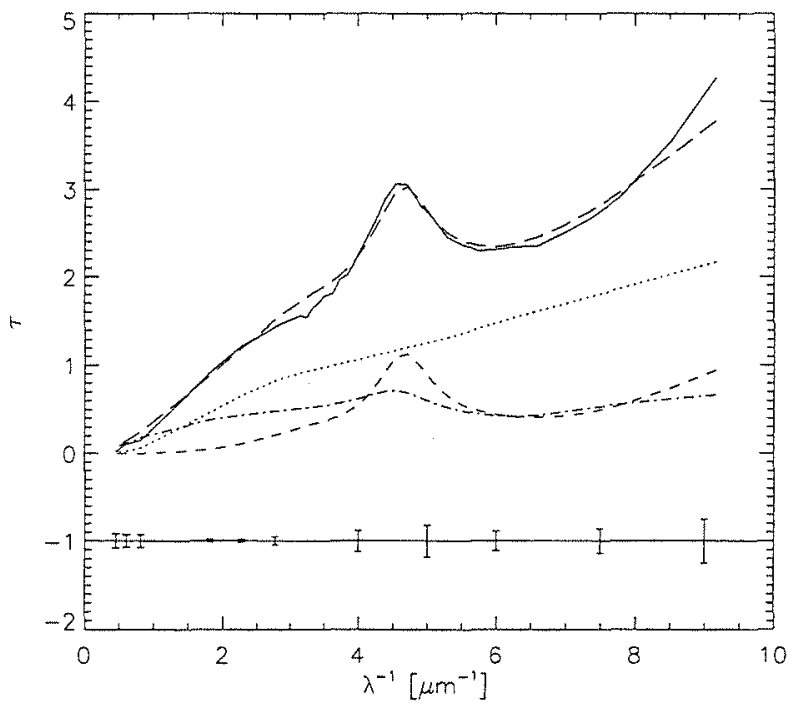

Fig. 9.- The fit to the extinction data (solid line) using the WD model (long dash line). The dotted, short dash, and dot-dash lines are the silicate, VSG, and larger graphitic components, respectively. Representative error bars are shown. 


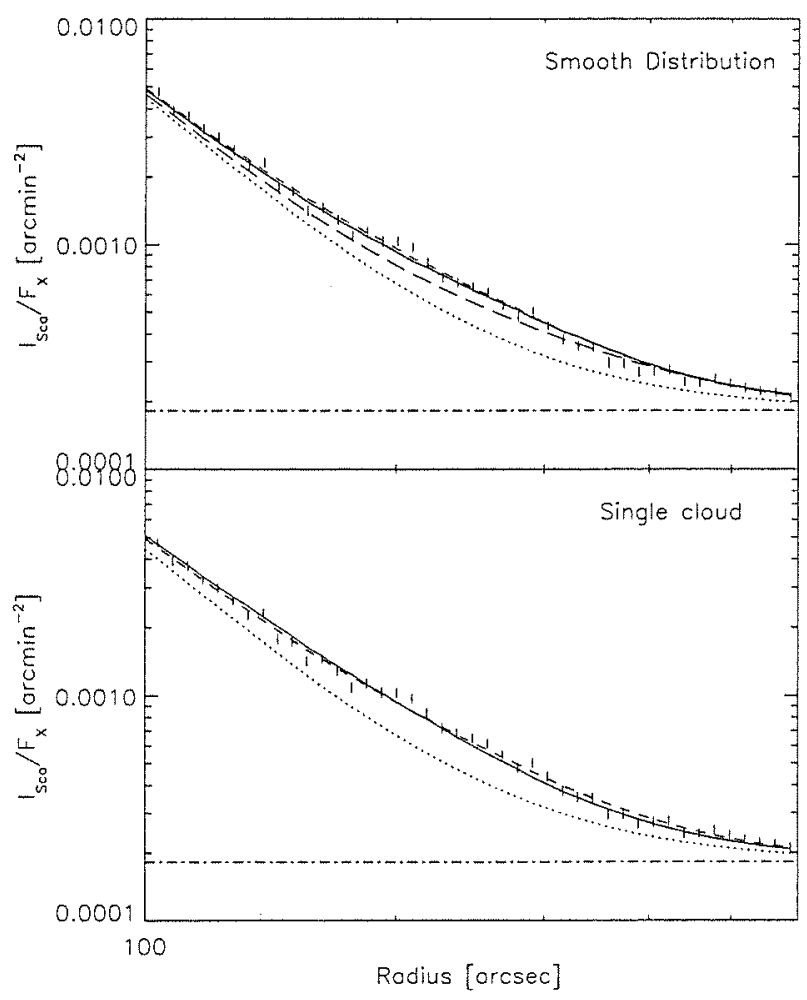

Fig. 10.- Best fits to the halo using the MRN model in a smooth dust distribution (top) and single cloud distribution (bottom). In both, the solid line corresponds to Set 1; short dash: Set 2; Dot-dash: background; Dotted line: PSF + background. For the top plot, long dash: the halo produced by assuming grain sizes and $N_{H}$ from the UV. 


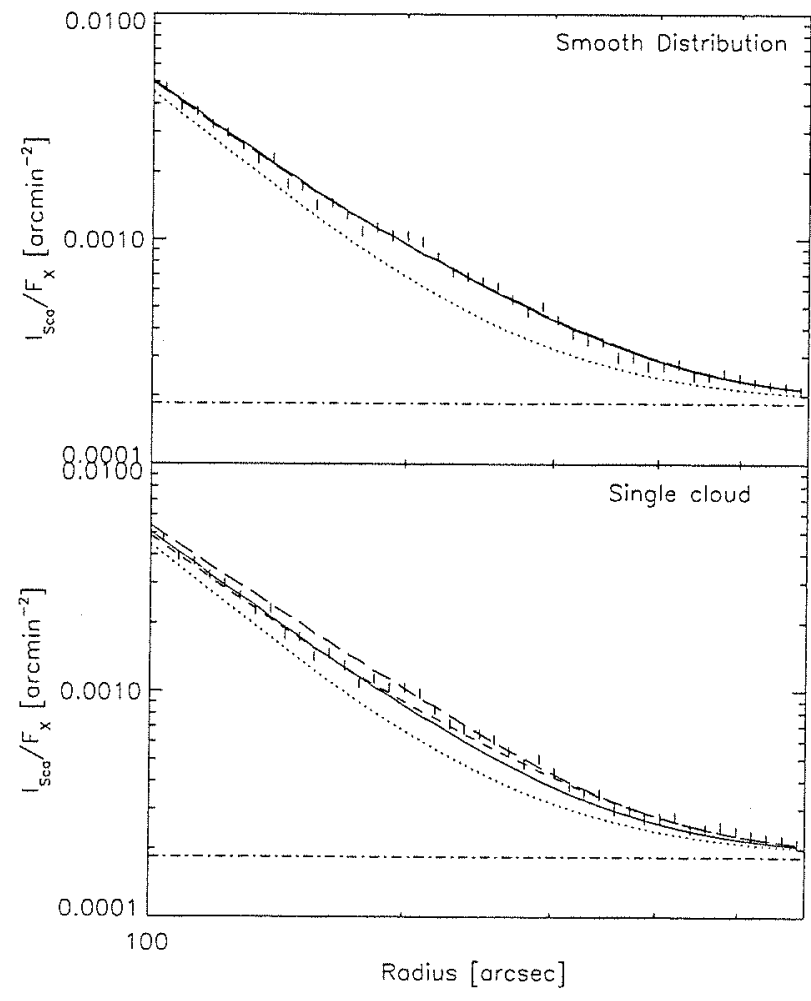

Fig. 11. - The best fit to the X-ray halo using the WD model in a smooth dust distribution (top) and single cloud distribution (bottom). For both, the solid line corresponds to Set 1; short dash: Set 2; Dot-dash: background; Dotted line: PSF + background. For the bottom plot, long dash: the halo produced by assuming grain sizes and $\mathrm{N}_{H}$ from the UV. 